\title{
Low Cycle Fatigue Life Estimation and Tracking for Industrial Gas Turbine Blades Using Fatigue Factor Approach
}

\author{
Ebigenibo Genuine Saturday', Thank-God Isaiah ${ }^{2}$ \\ ${ }^{1}$ Department of Mechanical Engineering, University of Port Harcourt, Port Harcourt, Nigeria \\ ${ }^{2}$ Department of Mechanical Engineering, Niger Delta University, Wilberforce Island, Nigeria \\ Email: satebi@yahoo.com
}

How to cite this paper: Saturday, E.G. and Isaiah, T. (2018) Low Cycle Fatigue Life Estimation and Tracking for Industrial Gas Turbine Blades Using Fatigue Factor Approach. Modern Mechanical Engineering, 8, 111-120.

https://doi.org/10.4236/mme.2018.82008

Received: February 5, 2018

Accepted: May 8, 2018

Published: May 11, 2018

Copyright $\odot 2018$ by authors and Scientific Research Publishing Inc. This work is licensed under the Creative Commons Attribution International License (CC BY 4.0).

http://creativecommons.org/licenses/by/4.0/

\begin{abstract}
Low cycle fatigue life consumption analysis was carried out in this work. Fatigue cycles accumulation method suitable even if engine is not often shut down was applied together with the modified universal sloped method for estimating fatigue cycles to failure. Damage summation rule was applied to estimate the fatigue damage accumulated over a given period of engine operation. The concept of fatigue factor which indicates how well engine is operated was introduced to make engine life tracking feasible. The developed fatigue life tracking method was incorporated in PYTHIA, Cranfield University in-house software and applied to 8 months of engine operation. The results obtained are similar to those of real engine operation. At a set power level, fatigue life decreases with increase in ambient temperature with the magnitude of decrease greater at higher power levels. The fatigue life tracking methodology developed could serve as a useful tool to engine operators.
\end{abstract}

\section{Keywords}

Damage Summation Rule, Fatigue Cycles Accumulation, Fatigue Factor, Fatigue Life Tracking, Low Cycle Fatigue

\section{Introduction}

The hot section components of gas turbines are prone to failure due to creep [1] [2] fatigue [3] [4] and creep-fatigue interaction [5] [6]. Despite the combined failure mode, creep failure and fatigue failure are often studied in isolation. This work centres on the estimation and tracking of low cycle fatigue life of industrial gas turbine blades. Low cycle fatigue life analysis involves the strain-life ap- 
proach to fatigue life analysis. Fatigue life consumption analysis of a component involves the determination of the number of cycles to failure at a given stress amplitude, and the number of stress cycles corresponding to a given block of load defined by the stress amplitude. The number of stress cycles corresponding to a given block of load involves stress cycle counting and different techniques can be employed [7] [8] [9] [10]. Considering the manner of operation of industrial gas turbines, a cycle counting model [11] which incorporates the frequent load variations is utilized in this work. The number of cycles to failure at a given load amplitude can be obtained using several methods. These include among others Morrow's equation, Smith-Watson-Topper relation, Universal slopes method, Mitchell's method and the modified universal slopes method [12] [13] [14] [15] [16]. In this work, the modified universal slopes method is adopted because it provides the best results in fitting fatigue data [15]. The target blade life is tracked by applying the damage summation rule provided by Palmgren and Miner [17].

Knowing that fatigue failure is stochastic in nature [18], obtaining an absolute value of fatigue failure from accumulated engine operation data will not be very appropriate. In the light of this, the time to fatigue failure obtained at a given period of engine operation is compared to that of a reference life where the reference life is estimated at a predefined point of engine operation. Fatigue factor, which is the ratio of the fatigue life obtained to the reference life is introduced in this work. This is similar to the concept of creep factor in [19] in creep life consumption analysis. Fatigue factor is a new concept introduced in this work for fatigue life consumption analysis in gas turbine blades. When the fatigue factor is less than 1, the engine was operated in worse condition but if greater than 1 , then engine operation is favourable (dictated mainly by the power level and variation of power level) compared to the reference condition. This concept will enable engine operators to have an idea of the nature of fatigue life consumption of the engine over a given period of engine operation even if the absolute life estimated is not completely accurate. A stress model was developed to estimate the stress at different points of engine operation. All the models developed and adopted in this work for blade fatigue life estimation and tracking are incorporated in PYTHIA [20], Cranfield University in-house gas turbine software, to make the life estimation and tracking feasible in a single platform by merely providing engine operation data. In this work, LM2500+ engine at Pulrose Power Station, Isle of Man was used as a case study. Details of this engine are in [21]. The fatigue life consumption of the gas producer turbine blades is considered in this work. The fatigue life of 8 different months of engine operation was estimated and tracked.

\section{Methodology}

The basic steps involved in estimating the fatigue life consumption of the gas producer turbine blades are estimation of the maximum stress at the 8 different sections of the blade at each point of engine operation/placing of the maximum 
stress into blocks, estimation of the cycles to failure corresponding to each block of maximum stresses, carrying out of damage summation to know the damage from the various blocks of stresses.

\subsection{Blade Stress Model}

The blade span is divided into 8 equal sections and the stresses due to centrifugal forces and the bending moment forces are estimated at the 8 different sections of the blade where the maximum value in the 8 sections is used for the fatigue life estimation. The stress model is also similar to that presented in [19]. At each section, the stresses are evaluated at the leading edge (LE), trailing edge (LE) and furthest point at the blade suction surface indicated as (SB). The centrifugal stress at each node $\sigma_{C, i}$ is given by Equation (1),

$$
\sigma_{C, i}=\frac{F_{i}}{A_{c s, i}}
$$

where $A_{c s, i}$ is the cross-sectional area of the blade at node $i$, and $F_{i}$ is the centrifugal force at node $i$.

The bending moment stresses at the base of each section and at the three locations is given by Equation (2),

$$
\sigma_{G, i}^{B M}=\frac{B M_{X X, i} \times Y_{G, i}}{I_{X X, i}}+\frac{B M_{Y Y, i} \times X_{G, i}}{I_{Y Y, i}}
$$

$\sigma_{G, i}^{B M}$ is the bending moment stress, $G$ stands for $L E$ at the leading edge, $T E$ at the trailing edge and $S B$ at the farthest point in the blade suction surface. $I_{X X}$ and $I_{Y Y}$ are the second moments of areas about the blade axial direction and tangential direction respectively. $X_{G, i}$ and $Y_{G, i}$ are the distances from the centre of gravity to the respective three locations in the axial and tangential directions respectively. The total stress $\sigma_{G, i}^{T o t}$ at each of the three locations at the base of each section of the blade is,

$$
\sigma_{G, i}^{T o t}=\sigma_{C, i}+\sigma_{G, i}^{B M}
$$

The overall maximum stress $\sigma_{\operatorname{Max}, i}$ at the base of all the sections considered is used for the life analysis of the blade.

\subsection{Cycle Counting Model}

The cycle counting model adopted in this work is that presented in [11]. Using this model, the number of stress cycles accumulated (termed equivalent stress cycles as the cycles are accumulated from different blocks of loads), $N_{e q}$ for a given period of engine operation is given by Equation (4),

$$
N_{e q}=\sum_{i=1}^{n}\left(\left|\frac{\sigma_{T o t, d}^{i+1}-\sigma_{T o t, d}^{i}}{\sigma_{\text {Tot, } d}^{S}}\right|\right)^{k}
$$

where $\sigma_{\text {Tot,d }}^{i}, \sigma_{\text {Tot, },}^{i+1}, \sigma_{\text {Tot,d }}^{S}$ and $k$ are the total stresses at each node for the $i^{\text {th }}$ data point, the next data point, at a set speed level and cycle determining exponent respectively. 


\subsection{Fatigue Life Estimation Model}

The modified universal slopes method [15] was applied in this work for estimating the fatigue cycles to failure at each maximum stress level estimated. This is given by Equation (5),

$$
\frac{\Delta \varepsilon}{2}=0.623\left(\frac{\sigma_{u}}{E}\right)^{0.832}\left(2 N_{f}\right)^{-0.09}+0.0196 \varepsilon_{f}^{0.155}\left(\frac{\sigma_{u}}{E}\right)^{-0.53}\left(2 N_{f}\right)^{-0.56}
$$

$\frac{\Delta \varepsilon}{2}$ is the total strain amplitude, $\sigma_{u}$ is the ultimate tensile strength of the material, $E$ is the Young's Modulus of the material, $\varepsilon_{f}$ is the true fracture ductility, and $N_{f}$ is the number of stress cycles to failure. Equation (5) is expressed in terms of nominal alternating stress amplitude, $\sigma_{a}$ as,

$$
\sigma_{a}=0.623 \sigma_{u}^{0.832} E^{0.168}\left(2 N_{f}\right)^{-0.09}+0.0196 \varepsilon_{f}^{0.155} \sigma_{u}^{-0.53} E^{1.53}\left(2 N_{f}\right)^{-0.56}
$$

The stress amplitude $\sigma_{a}$ is equivalent to the overall maximum stress $\sigma_{\text {Max }, i}$ obtained from the stress model. The number of stress cycles to failure $N_{f}$ is estimated from Equation (6) using the algorithm presented in Figure 1.

\subsection{Fatigue Life Estimation and Damage Accumulation}

When the number of fatigue cycles at each block of load is estimated and the numbers of cycles to failure corresponding to each block of load are obtained, the cumulative fatigue damage is estimated using damage summation rule given by Equation (6),

$$
D_{f, i}=\sum_{i=1}^{m} \frac{N_{e q, i}}{N_{f, i}}
$$

where $m$ is the number of blocks of load formed, $N_{e q, i}$ is the number of cycles corresponding to the $i^{\text {th }}$ block, $N_{f, i}$ is the number of cycles to failure corresponding to the stress amplitude at the $i^{\text {th }}$ block. $D_{f, i}$ is the fatigue damage parameter which is a fraction of the fatigue life consumed during the period of engine operation. For fatigue failure in practice, the damage parameter varies from 0.8 to 1.2 ; failure at unity damage parameter is assumed in this work. Thus, at the point of fatigue failure,

$$
\sum_{i=1}^{m} D_{f, i}=1
$$

The equivalent number of stress cycles could be estimated at any period of engine operation while the cycles to failure for a given block of loads in the given period is estimated using average stress level or the maximum stress in the block to be conservative. In any period of engine operation, the sum of the fatigue damage parameters, $D_{f, s}$ is given by Equation (8),

$$
D_{f, s}=\sum_{i=1}^{m} D_{f, i}=\sum_{i=1}^{m} \frac{\bar{N}_{a}}{N_{f, i}}
$$

where $\bar{N}_{a}$ is the mean value of the equivalent cycles accumulated for the entire period of engine operation, and $m$ is the total number of engine operation 


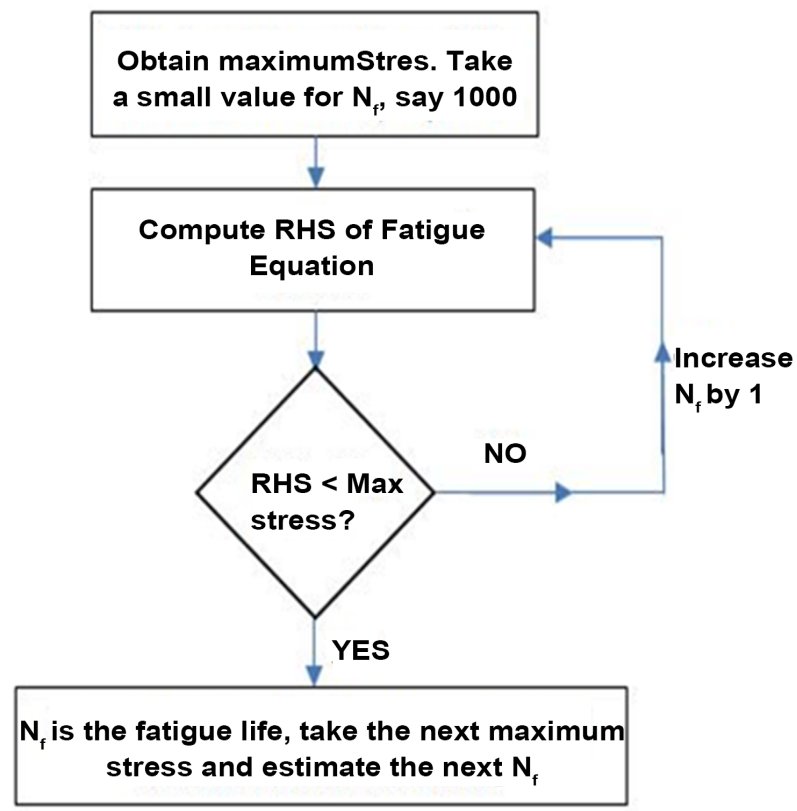

Figure 1. Algorithm for estimating fatigue cycles to failure.

points where fatigue damage parameters are evaluated. If $t_{a}=\sum_{i=1}^{m} t_{i}$ is the time taken to obtain $D_{f, s}$, the time to fatigue failure at any point of engine operation $t_{f, e q}$ is given as,

$$
t_{f, e q}=\frac{\sum_{i=1}^{m} t_{i}}{\sum_{i=1}^{m} \frac{\bar{N}_{a}}{N_{f, i}}}
$$

In terms of cycles to failure, the relation will be in the form,

$$
N_{f, e q}=\frac{\sum_{i=1}^{m} \bar{N}_{a}}{\sum_{i=1}^{m} \frac{\bar{N}_{a}}{N_{f, i}}}
$$

Results of fatigue life analysis are presented in terms of relative fatigue life analysis considered next.

\subsection{Relative Fatigue Life Analysis-The Concept of Fatigue Factor}

In the low cycle fatigue idealization, obtaining the equivalent number of fatigue cycles to failure at a given period of engine operation and estimating the fatigue damage parameter will only reveal the amount of fatigue life consumed, but this will not tell how well the engine is being operated for the period considered. Like the creep factor approach, if the number of cycles to failure is compared with the cycles to failure at a particular reference point, fatigue life will be obtained relative to the reference point, and this will give the operator of the engine an idea of the wellness of the operation of his engine. The fatigue factor (FF) is given by Equation (11), 


$$
\text { Fatigue Factor }(F F)=\frac{N_{f}}{N_{f, \operatorname{Re} f}}
$$

where $N_{f}$ is the number of cycles to failure at a given engine operation point (corresponding to a particular block of load), and $N_{f, \operatorname{Re} f}$ is the number of cycles to failure at the defined reference point. If $N_{f}<N_{f, \operatorname{Re} f}$, the engine is operated at a worse condition with respect to the reference point, if $N_{f}>N_{f, \operatorname{Re} f}$, the engine is operated at a favourable condition with respect to the reference condition. In a given period of engine operation, the equivalent fatigue factor will be estimated. This is the ratio of the equivalent fatigue life (equivalent cycles to fatigue failure) to the cycles to fatigue failure at the reference point; this is given by Equation (12),

$$
E F F=\frac{N_{f, e q}}{N_{f, \operatorname{Re} f}}
$$

$E F F$ is the equivalent fatigue factor, and $N_{f, e q}$ is the equivalent cycles to failure based on the entire period of engine operation.

\section{Fatigue Life Tracking and Results}

The fatigue life algorithm developed in this research is applied to 8 months of engine operation using real engine field data to ascertain the feasibility of blade life tracking process. The LM2500+ engine operated by Manx Utilities at Isle of Man was used as a case study and the power turbine blades were the target. Figure 2 shows the equivalent daily fatigue factors for the 8 months of engine operation considered while Figure 3 shows the monthly equivalent fatigue factors for each of the months of engine operation and the overall equivalent fatigue factor (indicated as OEFF). The effect of ambient temperature on fatigue life consumption at different shaft power levels was also investigated the results are presented in Figure 4. Here, ambient temperature was varied from $5^{\circ} \mathrm{C}$ to $30^{\circ} \mathrm{C}$, and shaft power levels of $70 \%$ to $100 \%$ were used.

The equivalent daily fatigue factors in Figures 2(a)-(h) are lower in January, February, March and December of engine operation compared to those obtained in June, July, August and November. This is because fatigue life depends on the stresses on the blades which arise from engine shaft speed and momentum changes. The latter depends on the amount of air intake. In January, February, March and December, lower ambient temperatures were recorded leading to higher air intake and hence higher alternating stress amplitude on the blades. The equivalent fatigue factor for each month of engine operation are thus lower in the months of January, February, March and December as in Figure 3. The overall equivalent fatigue factor (OEFF) is a little above unity, indicating that the fatigue life consumption of the blades is favourable in the 8 months of engine operation considered. The OEFF used is actually the equivalent fatigue life for the entire 8 months of engine operation.

At any given power level, increasing the ambient temperature through the range of temperatures considered lead to a decrease in the fatigue factor on the 


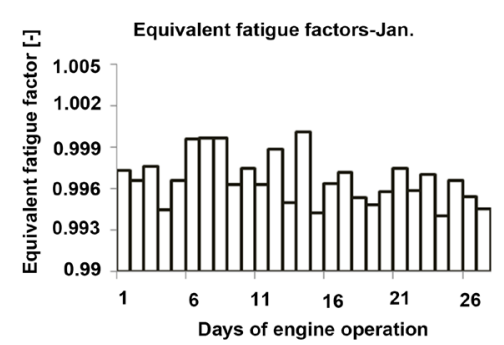

(a)

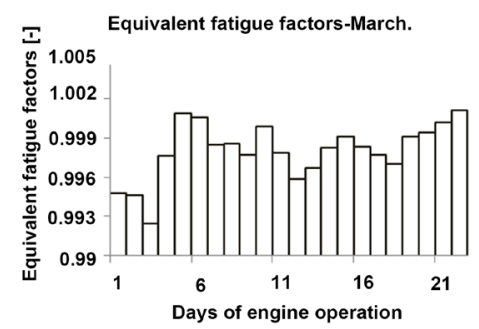

(c)

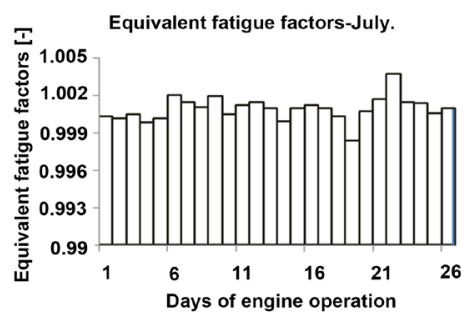

(e)

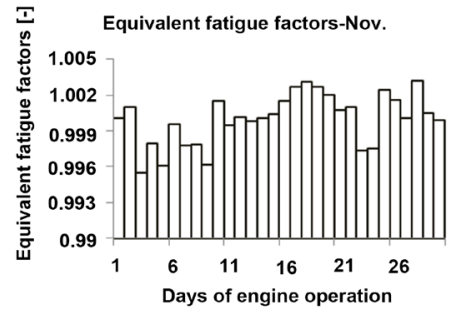

(g)

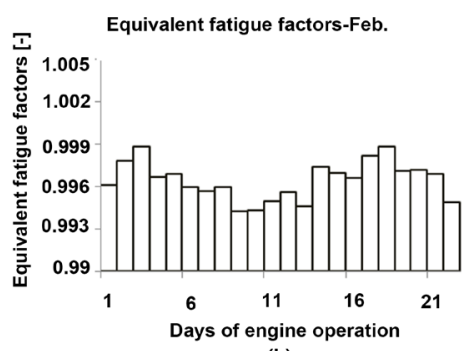

(b)

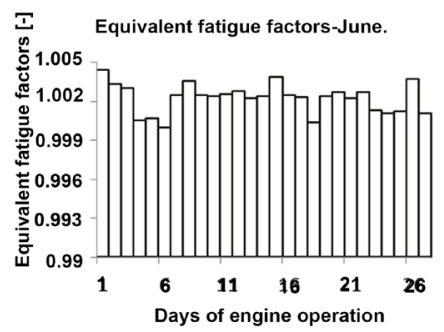

(d)

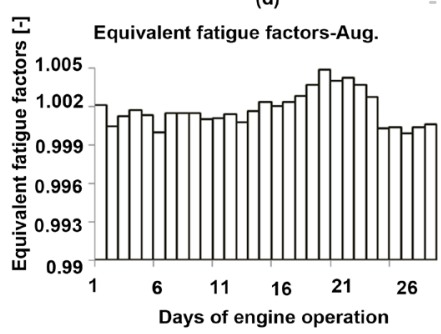

(f)

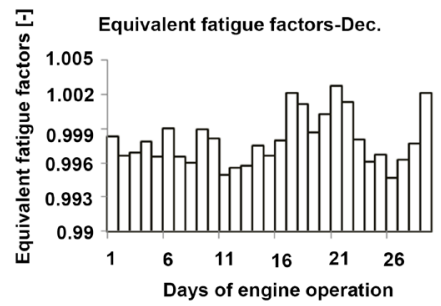

(h)

Figure 2. Equivalent fatigue factors for 8 months of engine operation.

average. The fatigue factors decrease with ambient temperature because increase in ambient temperature leads to lower mass intake. This is accompanied by lower momentum change stresses and tendency in shaft power level reduction. To keep the shaft power level constant, the engine speed increases leading to increase in centrifugal stresses. The increase in centrifugal stresses is more than the decrease in the momentum change stresses. This leads to reduction in fatigue life. The reduction in fatigue life increases with power level as higher stress amplitudes are experienced at higher power levels. This is shown in Figure 4.

\section{Conclusion}

Fatigue life consumption analysis and tracking methodology is developed in this work. The concept of fatigue factor is introduced which tells the wellness of engine operation and makes the engine life tracking feasible. The developed methodology was applied to 8 months of engine operation to track the fatigue life 


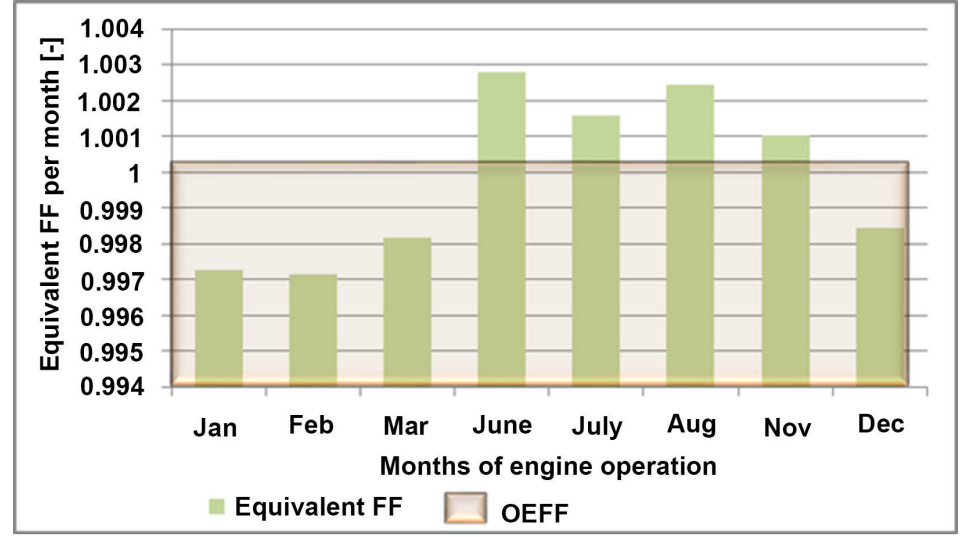

Figure 3. Equivalent fatigue factors for each month of engine operation.

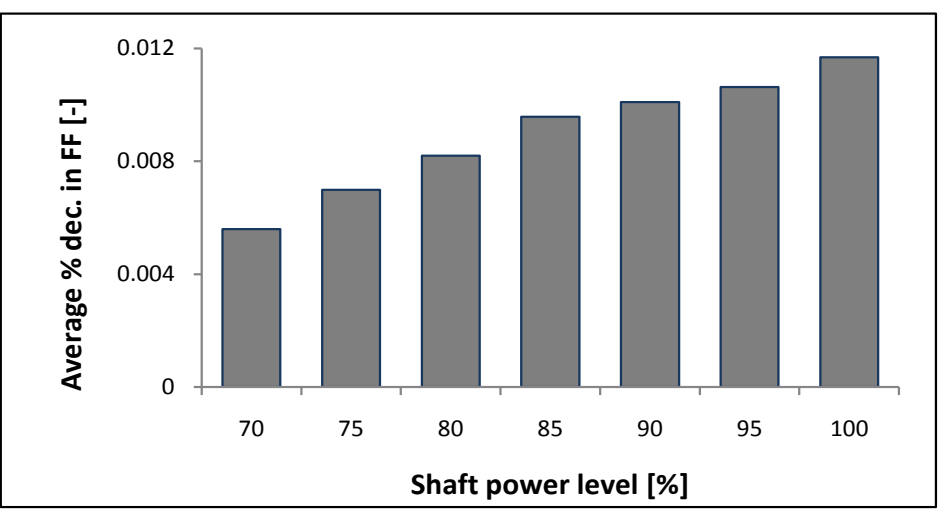

Figure 4. Average percentage decrease in fatigue factors with ambient temperature at different power levels.

consumption of the engine for the entire period, and to also test the feasibility of the life tracking process. The fatigue life consumption results obtained for the different months are in line with what is obtainable in real engine operations in many other works-higher fatigue life for lower power level operation and vice-versa. The effect of ambient temperature on fatigue life was investigated at different shaft power levels and two basic observations were made. Fatigue life decreases with increase in ambient temperature at a fixed shaft power level, and the value of the decrease in fatigue life is greater at higher power levels. The set power level is any power level in which the engine operates, say $80 \%$ or $900 \%$ of engine design power. The fatigue life tracking algorithm developed in this work could be used by engine operators in engine fatigue life consumption monitoring and hence aid them in their decision making pertaining to engine maintenance. Since gas turbine blade failure is hardly due to any single mode of component failure, creep-fatigue interaction failure need to be looked employing same relative life analysis methodology.

\section{Acknowledgements}

This work was supported by The Niger Delta Development Commission (NDDC) Nigeria, and Manx Utilities, Isle of Man, UK. 


\section{References}

[1] Cerri, G., Gazzino, M., Botta, F. and Salvini, C. (2008) Production Planning with Hot Section Life Prediction for Optimum Gas Turbine Management. International Journal of Gas Turbine, Propulsion and Power Systems, 2, 9-16.

[2] Nabarro, F.R.N. and de Villiers, F. (1995) The Physics of Creep and Creep-Resistant Alloys. CRC Press, Taylor \& Francis Group, UK.

[3] Harmon, D., Mcclure, M., Grelotti, R. and Hartford, E. (2010) Unified Low Cycle Fatigue for Gas Turbine Engine Rotor Alloys. Proceedings of 51 st AIAA/ASME/ ASCE/AHS/ASC Structures, Structural Dynamics, and Materilas Conference, Orlando, 12-15 April, 1-10.

[4] Nicholas, T. (1999) Material Allowances for High Cycle Fatigue in Gas Turbine Engines. RTO AVT Specialists Meeting on Application of Damage Tolerance Principles for Improved Airworthiness of Rotorcraft, 1-9.

[5] Mao, H. and Mahadevan, S. (2000) Creep Fatigue Reliability of High Temperature Materials. 8th ASCE Specialty Conference on Probabilistic Mechanics and Structural Reliability, University of Notre Dame, 4-26 July, 1-6.

[6] Villasante, M. (2011) Predictive Methods for Combined Cycle Fatigue in Gas Turbine Blades. Aerodays, Madrid, 30 March-1 April 2011, 1-8.

[7] Downing, S. and Socie, D. (1982) Simple Rainflow Counting Algorithms. International Journal of Fatigue, 31-40. https://doi.org/10.1016/0142-1123(82)90018-4

[8] Glinka, G. and Kam, J. (1987) Rainflow Counting Algorithm for Very Long Stress Histories. International Journal of Fatigue, 9, 223-228. https://doi.org/10.1016/0142-1123(87)90025-9

[9] Hong, N. (1991) A Modified Rainflow Counting Method. International Journal of Fatigue, 13, 465-469. https://doi.org/10.1016/0142-1123(91)90481-D

[10] Baek, S.H., Cho, S.S. and Joo, W.S. (2008) Fatigue Life Prediction Based on the Rainflow Cycle Counting Method for the End Beam of a Freight Car Bogie. International Journal of Automotive Technology, 9, 95-101. https://doi.org/10.1007/s12239-008-0012-y

[11] Saturday, E.G. (2017) Development and Application of Fatigue Cycles Accumulation Model for Industrial Gas Turbines Operation. Journal of Emerging Trends in Engineering and Applied Sciences, 8, 30-36.

[12] Kim, K.S., Chen, X., Han, C. and Lee, H.W. (2002) Estimation Methods for Fatigue Properties of Steels under Axial and Torsional Loading. International Journal of Fatigue, 24, 783-793. https://doi.org/10.1016/S0142-1123(01)00190-6

[13] Ince, A. and Glinka, G. (2011) A Modification of Morrow and Smith-WatsonTopper Mean Stress Correction Models. Fatigue \& Fracture of Engineering Materials \& Structures, 34, 854-867. https://doi.org/10.1111/j.1460-2695.2011.01577.x

[14] Dowling, N.E. (2004) Mean Stress Effects in Stress-Life and Strain-Life Fatigue. Soc. Automot. Eng. Inc.

[15] Park, J. and Song, J. (1995) Detailed Evaluation of Methods for Estimation of Fatigue Properties. International Journal of Fatigue, 17, 365-373. https://doi.org/10.1016/0142-1123(95)99737-U

[16] Suresh, S. (2004) Fatigue of Materials. 2nd Edition, Cambridge University Press, New York.

[17] Hashin, Z. (1979) A Reinterpretation of Palmgren-Miner Rule for Fatigue Life Prediction. Office of Naval Research, Arlington.

[18] Sapsard, M. (2000) Recommended Practices for Monitoring Gas Turbine Engine 
Life Consumption. RTO/NATO-NASA, Canada Communication Group Inc.

[19] Abdul Ghafir, M.F., Li, Y.G., Singh, R., Huang, K. and Feng, X. (2010) Impact of Operating and Health Conditions on Aero Gas Turbine Hot Section Creep Life using a Creep Factor Approach. Proceedings of ASME Turbo Expo 2010. Power for Land, Sea and Air, 14-16 June 2010, 1-13.

[20] Li, Y.G. and Singh, R. (2005) An Advanced Gas Turbine Gas Path Diagnostic System-PYTHIA. The XVII International Symposium on Air Breathing Engines, Munich, 1-12.

[21] Saturday, E.G., Li, Y.G., Ogiriki, E.A. and Newby, M.A. (2017) Creep-Life Usage Analysis and Tracking for Industrial Gas Turbines. Journal of Propulsion and Power, 33, 1305-1314. https://doi.org/10.2514/1.B35912 